Contents available at: Sri Lanka Journals Online

\title{
Study on Trans Fat Content of Selected Foods Commercially Available in Colombo District of Sri Lanka
}

M.L.D. Wasana ${ }^{1}$, A. de Silva ${ }^{2}$, N. Gunawardana ${ }^{3}$, D.C.K. Illeperuma ${ }^{4}$, W.M.P.B.Weerasinghe ${ }^{5}$, W.M.D.C Weerathunga ${ }^{5}$, S. Ekanayake6, T. Madhujith ${ }^{4}$

1 Postgraduate Institute of Agriculture, University of Peradeniya, Peradeniya, 20400, Sri Lanka

${ }^{2}$ WHO Regional Office for South-East Asia, New Delhi, India

3 World Health Organization, Country Office for Sri Lanka, Colombo 05, Sri Lanka

${ }^{4}$ Department Food Science and Technology, Faculty of Agriculture, University of Peradeniya, Peradeniya, 20400, Sri Lanka

${ }^{5}$ Veterinary Research Institute, Gannoruwa, Sri Lanka

${ }^{6}$ Department of Biochemistry, Faculty of Medical Sciences, University of Sri Jayawardhanapura, Nugegoda, Sri Lanka

\section{ARTICLE INFO}

\section{Article history:}

Received: 24 August 2020

Revised version received: 18 October 2020

Accepted: 5 November 2020

Available online: 01 October 2021

\section{Keywords:}

Monounsaturated fatty acids

Polyunsaturated fatty acids

Saturated fatty acids

Total fat

Trans fat

\section{Citation:}

Wasana, M.L.D., de Silva, A., Gunawardana, N., lleperuma, D.C.K.I,.Weerasinghe, W,M.P.B, Weerathunga, W.M.D.C, Ekanayake, S. and Madhujith, T. 2021. Study on trans fat content of selected foods commercially available in Colombo District, of Sri Lanka. Tropical Agricultural Research, 32(4): 471-479.

DOI: http://doi.org/10.4038/tar.v32i4.8515

Wasana, M.L.D.

https://orcid.org/0000-0002-3106-8740

\section{ABSTRACT}

The relationship between the dietary consumption of unsaturated fatty acids with trans configuration and increased risk of coronary heart diseases, cancer, diabetes mellitus among others is well established. The sources of intake of trans fats mainly include foods produced using partially hydrogenated oils, fried snacks and baked goods. Fried and baked foods available at eateries, restaurants and sold by roadside vendors are very popular among Sri Lankans. Moreover, home-made fried foods are regularly consumed in the country. Furthermore, reuse of frying oils also contributes to generation of trans fats. Therefore, fried foods are suspected to contain high quantities of trans fat. The present study was designed to quantify the trans fat level of selected processed foods collected from Colombo district. The total fat (saturated fatty acids (SFA), monounsaturated fatty acids (MUFA), polyunsaturated fatty acids (PUFA) and unsaturated fatty acids (UFA)) and trans fatty acid (TFA) contents of food samples were analyzed using GLC. Trans fat content ranged from $0.00-1.50 \mathrm{~g} / 100 \mathrm{~g}$ in food samples tested. The highest trans fat content was observed in chilli paste samples. Fried rice, collected from Colombo district also contained $0.91 \mathrm{~g} / 100 \mathrm{~g}$ of food. It was revealed that the other food items contain <1g / $100 \mathrm{~g}$ of trans fatty acid.

* Corresponding author: tmadujith@agri.pdn.ac.lk 


\section{INTRODUCTION}

The relationship between the dietary consumption of trans fatty acids with increased risk of coronary heart diseases, cancer and diabetes mellitus has been reported (De Souza et al., 2015). According to the Ministry of Health, Nutrition and Indigenous Medicine, Sri Lanka, ischemic heart diseases are the leading cause of death in Sri Lanka since 1995 (Madhujith and Sivakanthan, 2020). The main sources of trans fats include foods produced using partially hydrogenated oils, fried snacks and baked goods. All-natural fatty acids present in foods are in the cis form while some of the cis fatty acids are transformed into trans fatty acids during processing. With revelation of the adverse health effects associated with trans-fat consumption many western countries implemented strict regulations aiming at reducing trans fats in their food supply. Identifying the risk associated with trans fats, the United States Food and Drug Administration (US-FDA) implemented strict legislations to reduce trans fats in 2006 (Kavanagh et al., 2007) and subsequently many other countries also formulated their national legislations restricting the trans fat content in their food supply (Wanders et al., 2017). However, in Sri Lanka, currently there are no legislations to regulate trans fat levels in foods. Besides, the public awareness of the trans fat levels of foods and its health consequences is lacking.

Fried and baked foods available at various outlets such as eateries, restaurants, and roadside kiosks as well as home-made fried foods are consumed widely by Sri Lankans of all ages. Thus, consumption of fried foods and baked foods could have a major contribution for the trans fat in daily diet, which, in turn contributes to increased risk of coronary heart diseases.

Frying is a process whereby food is completely immersed and held in hot frying fat (Tynek et al., 2001). Among the sources of human intake of trans fats, deep fried foods have been identified to be one of the most important sources of trans fats in several countries. Deep frying is widely used by the restaurants, street vendors, confectioners, bakers as well as at domestic level (Rani et al., 2010). Fried foods are preferred by consumers all over the world due to their unique sensory qualities such as crispy texture, aroma and golden brown color (Aladedunye and Przybylski, 2009). However, during the frying process trans fats are generated especially when unsaturated oil is used for frying. Frying foods using oils containing polyunsaturated fatty acids at temperatures above $180{ }^{\circ} \mathrm{C}$ for prolonged period of time generates trans fats. The extent of formation of trans fat during frying depends on the temperature and duration of frying (Martinet al., 2007). Furthermore, the reuse of frying oils many times for frying snacks such as rolls, cutlets and some foods of Indian origin such as Samosa and Wadei leads to generation of significant quantities of trans fats which are subsequently absorbed by the fried products. Meanwhile, it has been observed that the chemical reactions that take place during deep-fat frying are different from those during continuous heating. Besides, different oils have been found to behave differently regarding the rate of formation of polar components and secondary oxidized products (Aladedunye and Przybylski, 2009). Furthermore, it is suspected that some organized groups collect spent oils that are removed after use from restaurants and reuse them.

Due to the evidences of adverse health effects of trans fats, many developed countries have adopted alternative approaches for partial hydrogenation such as the use of structured oils (Kaushik and Grewal, 2017; Remig et al., 2010). Since fried foods and bakery food items are suspected to be one of the major sources of trans fat in Sri Lankan diet, the present study was designed to quantify the total and trans fatty acid content and fatty acid groups, namely saturated, mono unsaturated and polyunsaturated fatty acid content of processed food products which are highly consumed by Sri Lankans.

\section{METHODOLOGY}

\section{Sampling procedure}

A total of 30 food samples were collected from bakery chains, restaurants, grocery stores and small food outlets in Colombo district representing all ethnicities and dietary variations. The number of samples was decided based on the number required to capture the expected variation of the foods and the feasibility of laboratory analysis and related costs.

\section{Preparation of composite samples}

The collected samples were placed in clear polythene bags, tagged, placed in insulated boxes and transported to the analytical laboratory under cold conditions with minimum delay. The Composite samples were prepared by taking the entire contents from each of packages, combining the contents and homogenizing. A subsample was taken from resulting composite sample for 
extraction of fat. The reminder of the composite was stored for future use under frozen conditions.

\section{Extraction of lipid from food samples}

The food samples were extracted and analyzed according to the method described in Ratnayake, 2004. The food samples were dipped in liquid nitrogen and then ground using mortar and pestle to obtain a fine powder. Ground food samples were stored in amber colored glass vials under frozen conditions until use. The sample was accurately weighed and placed in screw-capped glass test tubes $(30 \mathrm{~mL})$. Ethanol $(2 \mathrm{~mL}), 10 \mathrm{~mL}$ of $8.3 \mathrm{M} \mathrm{HCl}$ were added into the test tubes and mixed well. Test tubes containing the samples were heated in a water bath (WBB7-45, Memmert, Germany) for 60 min at $80{ }^{\circ} \mathrm{C}$. The content of the test tube was vortexed every $10 \mathrm{~min}$ to incorporate any particulates adhered on to the walls of the test tube into solution. After $60 \mathrm{~min}$ of heating, the test tubes were removed, allowed to cool to room temperature $\left(27{ }^{\circ} \mathrm{C}\right)$ and mixed with $2.0 \mathrm{~mL}$ ethanol and $5.0 \mathrm{~mL}$ diethyl ether. The contents of the tube were transferred into a $500 \mathrm{~mL}$ separatory funnel. To ensure quantitative transfer, the tube was washed with a mixture of $10 \mathrm{~mL}$ diethyl ether and $10 \mathrm{~mL}$ hexane. Rinsing was repeated with the same solvent mixture. These rinses were transferred to the separatory funnel and mixed briefly. Diethyl ether $(50 \mathrm{~mL})$ and $50 \mathrm{~mL}$ of hexane were added to the separatory funnel, mixed briefly and the layers were allowed to separate. The contents were allowed to settle at least $1 \mathrm{~h}$ until the upper layer turned clear. The upper organic layer was slowly decanted into a $500 \mathrm{~mL}$ Erlenmeyer flask with a glass stopper. Then $10 \mathrm{ml}$ of diethyl ether and $25 \mathrm{~mL}$ of hexane were added to the 500 $\mathrm{mL}$ separatory funnel containing the bottom layer (aqueous layer). The contents were mixed thoroughly and allowed the layers to separate. The top organic layer was slowly decanted into the 500 $\mathrm{mL}$ Erlenmeyer flask containing the previously collected organic layer and the aqueous layer was discarded. The combined organic layers were dried by passing through a bed of anhydrous sodium sulphate. The dried organic layer was filtered into a round bottom flask and the solvent (diethyl ether + hexane) was evaporated, in vacuo. The residue remaining in the round bottom flask contains extracted fat.

\section{Methylation of extracted fat}

The extracted fat was dissolved in $2 \mathrm{ml}$ toluene in a screw-capped glass test tube $(20 \mathrm{~mL})$. Then $2 \mathrm{~mL}$ of $7 \% \mathrm{BF}_{3}$-methanol reagent was added and the vials were capped. The tube was heated at $100{ }^{\circ} \mathrm{C}$ for 45 min in a heating block and the tube was gently shaken every 10 min during heating. The tubes were removed from the heating block, and allowed to cool to room temperature and then $5 \mathrm{~mL}$ of distilled water, $2 \mathrm{~mL}$ of hexane and $1 \mathrm{~g}$ of sodium sulphate were added. The tubes were capped and shaken. After 10 min, FAME- hexane solution was collected into small glass vials. Then the vial was flushed with nitrogen and capped. The samples were analyzed immediately by gas liquid chromatograph (GLC) (Master GC, Dani Instruments, Italy).

\section{Analysis of fatty acids using gas chromatographic analysis}

Prepared FAMEs were analyzed by GLC using a 100 $\mathrm{m}$ fused silica capillary column. The operating parameters used were injection port temperature $250{ }^{\circ} \mathrm{C}$, detector temperature $250{ }^{\circ} \mathrm{C}$ and oven temperature $180^{\circ} \mathrm{C}$. The carrier gas was hydrogen with; column head pressure of $170 \mathrm{kPa}(25 \mathrm{psi})$; flow rate of $1.0 \mathrm{~mL} / \mathrm{min}$; linear velocity of $26 \mathrm{~cm} / \mathrm{s}$; split ratio of 100:1. The injection volume was $1 \mu \mathrm{L}$. The column and GLC performance were checked using a Supelco 37 FAME mixture covering the entire range of fatty acid under investigation.

GLC peaks were identified by their retention times. Total fat content was calculated as sum of individual fatty acids. Individual fatty acids (including all trans fatty acid isomers) were expressed. Trans fatty acid composition was calculated and expressed both as percent total fatty acids and as g fatty acids per $100 \mathrm{~g}$ of test food sample.

The fatty acid composition of samples were statistically analyzed by comparing the mean by Duncan's multiple range test.

\section{RESULTS AND DISCUSSION}

Thirty food items from Colombo district were analyzed for total fat content, trans fatty acids (TFA), saturated fatty acids (SFA), monounsaturated fatty acids (MUFA), polyunsaturated fatty acids (PUFA) and unsaturated fatty acids (UFA).

It was revealed that chilli paste contained the highest quantity of trans fatty acid which is equivalent to $1.57 \mathrm{~g} / 100 \mathrm{~g}$ of fat. Manioc chips also contained $0.95 \mathrm{~g}$ of TFA in $100 \mathrm{~g}$ of food products. All other food products contained less than $1 \mathrm{~g}$ of trans fatty acid in $100 \mathrm{~g}$ of food (Tables 1 and 2). 
Table 1. Mean $( \pm S D, n=3)$ total fat and trans fatty acid (TFA) contents of foods collected from restaurants and bakeries.

\begin{tabular}{lccc}
\hline Food & Total Fat $\mathbf{~ g / 1 0 0 ~} \mathbf{g}$ food) & TFA $(\mathbf{g} / \mathbf{1 0 0} \mathbf{g}$ of fat) & TFA $\mathbf{g} / \mathbf{1 0 0} \mathbf{~ g ~ f o o d})$ \\
\hline Fried whole chicken & $18.60( \pm 0.2)$ & $0.45( \pm 0.0)$ & $0.08( \pm 0.0)$ \\
Chicken nuggets & $7.81( \pm 0.05)$ & $0.27( \pm 0.0)$ & $0.02( \pm 0.0)$ \\
Fried whole fish & $10.10( \pm 0.1)$ & $0.64( \pm 0.0)$ & $0.06( \pm 0.0)$ \\
Chilli paste & $40.00( \pm 0.2)$ & $3.92( \pm 0.0)$ & $1.57( \pm 0.0)$ \\
French fries & $14.22( \pm 0.1)$ & $0.24( \pm 0.0)$ & $0.03( \pm 0.0)$ \\
Manioc chips & $23.33( \pm 0.2)$ & $4.06( \pm 0.0)$ & $0.95( \pm 0.0)$ \\
Popcorn & $18.00( \pm 0.1)$ & $2.04( \pm 0.0)$ & $0.37( \pm 0.0)$ \\
Fried rice & $7.48( \pm 0.04)$ & $0.00( \pm 0.0)$ & $0.00( \pm 0.0)$ \\
Kottu & $4.89( \pm 0.01)$ & $0.00( \pm 0.0)$ & $0.00( \pm 0.0)$ \\
Pizza & $3.61( \pm 0.02)$ & $0.00( \pm 0.0)$ & $0.00( \pm 0.0)$ \\
White bread & $5.02( \pm 0.05)$ & $0.00( \pm 0.0)$ & $0.00( \pm 0.0)$ \\
Sandwich bread & $5.13( \pm 0.05)$ & $0.00( \pm 0.0)$ & $0.00( \pm 0.0)$ \\
Parata & $4.61( \pm 0.02)$ & $0.00( \pm 0.0)$ & $0.00( \pm 0.0)$ \\
Poori & $11.64( \pm 0.1)$ & $0.00( \pm 0.0)$ & $0.00( \pm 0.0)$ \\
\hline
\end{tabular}

Table 2. Mean $( \pm S D, n=3)$ of total fat and trans fatty acid (TFA) content of selected street foods

\begin{tabular}{lccc}
\hline Food & Total Fat $\mathbf{( g / 1 0 0} \mathbf{g}$ food) & TFA $(\mathbf{g} / \mathbf{1 0 0} \mathbf{g}$ of fat) & TFA (g/100 g food) \\
\hline Fried rice & $4.85( \pm 0.01)$ & $0.00( \pm 0.0)$ & $0.00( \pm 0.0)$ \\
Kottu & $5.86( \pm 0.05)$ & $0.00( \pm 0.0)$ & $0.00( \pm 0.0)$ \\
Cutlets & $4.93( \pm 0.03)$ & $0.00( \pm 0.0)$ & $0.00( \pm 0.0)$ \\
Rolls & $12.65( \pm 0.05)$ & $0.00( \pm 0.0)$ & $0.00( \pm 0.0)$ \\
dhal wade & $10.33( \pm 0.1)$ & $0.00( \pm 0.0)$ & $0.00( \pm 0.0)$ \\
Ulundu wade & $12.11( \pm 0.2)$ & $0.00( \pm 0.0)$ & $0.00( \pm 0.0)$ \\
Prawn wade & $17.51( \pm 0.1)$ & $0.00( \pm 0.0)$ & $0.00( \pm 0.0)$ \\
Samosa & $10.75( \pm 0.3)$ & $1.36( \pm 0.0)$ & $0.15( \pm 0.0)$ \\
Patties & $5.68( \pm 0.2)$ & $1.62( \pm 0.0)$ & $0.09( \pm 0.0)$ \\
Curry rotti & $5.65( \pm 0.1)$ & $1.93( \pm 0.0)$ & $0.11( \pm 0.0)$ \\
Murukku & $26.25( \pm 0.1)$ & $0.57 \pm \pm 0.0)$ & $0.15( \pm 0.0)$ \\
Manioc chips & $24.06( \pm 0.2)$ & $0.69( \pm 0.0)$ & $0.17( \pm 0.0)$ \\
Dodol & $9.63( \pm 0.2)$ & $0.00( \pm 0.0)$ & $0.00( \pm 0.0)$ \\
Unduwel & $20.01( \pm 0.1)$ & $0.00( \pm 0.0)$ & $0.00( \pm 0.0)$ \\
Kadala & $7.23( \pm 0.05)$ & $1.01( \pm 0.0)$ & $0.07( \pm 0.0)$ \\
Popcorn & $1.83( \pm 0.01)$ & $0.89( \pm 0.0)$ & $0.02( \pm 0.0)$ \\
\hline
\end{tabular}

Food items collected from restaurants and bakeries contained higher amounts of trans fats than food items collected from roadside vendors. Table 01 and 02 show the total fat content and trans fat content of foods collected from restaurants/ bakeries and street vendors. Total fat content, saturated fatty acids (SFA), monounsaturated fatty acids (MUFA), polyunsaturated fatty acids (PUFA) and unsaturated fatty acids (UFA) content were analyzed in selected restaurant and street food items. The total fat content of processed foods from bakery and restaurant foods varied from 3.6 to $40 \%$. The highest total fat content was observed in chilli paste while the lowest quantity was observed in pizza. Among the foods collected from street shops, total fat content varied from $1.8 \%$ (popcorn) to $26.3 \%$ (Murukku).

Among restaurant and bakery food category, UFA content of food was higher than the SFA content. The SFA content varied from 36.9\% (sandwich bread) to $57.7 \%$ (pizza). Pizza showed the lowest UFA content of $42.3 \%$ while the highest UFA quantity of was recorded in fried whole chicken (64.1\%) (Table 3). Among street foods, the SFA content ranged between $20.1 \%$ (patties) and $77.4 \%$ (Dodol) (Table 04). Dodol is a traditional Sri Lankan dessert prepared from coconut fat, therefore it contains high level of saturates. Coconut oil contains $>90 \%$ of SFA (Bhatnagar et al., 2009).This is the reason for having a very high 
content of SFA in Dodol. Most of the fried foods such as rolls, cutlets, Wadei contain fairly high amounts of SFA due to the use of palm oil for frying of them.

Elaidic acid (C18:1 trans-9) and linolelaidic acid (C18:2 trans-6) were identified as the major trans fatty acids in food items analyzed. Elaidic and linolelaidic acids are the major trans fatty acids generated during processing such as partial hydrogenation and frying. Partial hydrogenation of unsaturated oils is the process that generates high quantities of trans fat. Although the world leading margarine, shortenings and fat spread manufacturers started using alternative methods for partial hydrogenation it is speculated that still some manufacturers continue to use partial hydrogenation. These fat based products are commonly used as ingredients in bakery industry, therefore, it can be assumed that bakery food items such as cake, muffins, pastries, cream buns may potentially contain high amounts of trans fat.

Table 3. Mean $( \pm S D, n=3)$ of different fatty acid groups of selected foods collected from restaurants and bakeries

\begin{tabular}{lcccc}
\hline Food item & $\begin{array}{c}\text { SFA } \\
\text { (\% from total } \\
\text { fat) }\end{array}$ & $\begin{array}{c}\text { MUFA } \\
\text { (\% from total } \\
\text { fat) }\end{array}$ & $\begin{array}{c}\text { PUFA } \\
\text { (\% from total } \\
\text { fat) }\end{array}$ & $\begin{array}{c}\text { UFA } \\
\text { (\%om total } \\
\text { fat) }\end{array}$ \\
\hline Fried whole chicken & $35.95( \pm 0.1)$ & $4.93( \pm 0.05)$ & $59.13( \pm 0.5)$ & $64.06(0.3)$ \\
Chicken nuggets & $38.72( \pm 0.1)$ & $2.67( \pm 0.01)$ & $58.61( \pm 0.2)$ & $61.28(0.04)$ \\
Fried whole fish & $42.00( \pm 0.2)$ & $4.90( \pm 0.02)$ & $53.09( \pm 0.3)$ & $57.99( \pm 0.6)$ \\
Chilli paste & $48.28( \pm 0.1)$ & $11.06( \pm 0.1)$ & $40.67( \pm 0.1)$ & $51.73( \pm 0.3)$ \\
French fries & $44.44( \pm 0.2)$ & $2.90( \pm 0.05)$ & $52.66( \pm 0.2)$ & $55.56( \pm 0.05)$ \\
Manioc chips & $38.96( \pm 0.3)$ & $6.46( \pm 0.02)$ & $54.58( \pm 0.3)$ & $61.04( \pm 0.2)$ \\
Popcorn & $57.67( \pm 0.2)$ & $13.09( \pm 0.1)$ & $29.24( \pm 0.1)$ & $42.33( \pm 0.1)$ \\
Fried rice & $43.81( \pm 0.35)$ & $5.31( \pm 0.2)$ & $50.88( \pm 0.1)$ & $56.19( \pm 0.2)$ \\
Kottu & $41.91( \pm 0.1)$ & $5.36( \pm 0.1)$ & $52.73( \pm 0.2)$ & $58.09( \pm 0.2)$ \\
Pizza & $57.72( \pm 0.2)$ & $4.62( \pm 0.01)$ & $37.67( \pm 0.1)$ & $42.29( \pm 0.1)$ \\
White bread & $37.45( \pm 0.3)$ & $12.23( \pm 0.2)$ & $50.3( \pm 0.3)$ & $62.53( \pm 0.2)$ \\
Sandwich bread & $36.94( \pm 0.1)$ & $10.40( \pm 0.1)$ & $52.65( \pm 0.2)$ & $63.05( \pm 0.1)$ \\
Parata & $51.32( \pm 0.3)$ & $14.84( \pm 0.1)$ & $33.84( \pm 0.2)$ & $48.68( \pm 0.2)$ \\
Poori & $39.44( \pm 0.1)$ & $12.75( \pm 0.2)$ & $47.81( \pm 0.2)$ & $60.56( \pm 0.2)$ \\
\hline
\end{tabular}

SFA - Saturated fatty acid; MUFA - Monounsaturated fatty acid; PUFA - Polyunsaturated fatty acid; UFA - Unsaturated fatty acid.

Table 4. Mean $( \pm S D, n=3)$ of different fatty acid groups of selected street foods

\begin{tabular}{lcccc}
\hline Food item & $\begin{array}{c}\text { SFA } \\
\text { (\% from total fat) }\end{array}$ & $\begin{array}{c}\text { MUFA } \\
\text { (\% from total fat) }\end{array}$ & $\begin{array}{c}\text { PUFA } \\
\text { (\% from total fat) }\end{array}$ & $\begin{array}{c}\text { UFA } \\
\text { (\% from total fat) }\end{array}$ \\
\hline Fried rice & $43.81( \pm 0.3)$ & $5.31( \pm 0.05)$ & $50.88( \pm 0.3)$ & $56.19( \pm 0.25)$ \\
Kottu & $41.91( \pm 0.2)$ & $5.36( \pm 0.02)$ & $52.73( \pm 0.1)$ & $58.09( \pm 0.15)$ \\
Cutlets & $52.03( \pm 0.1)$ & $0.38( \pm 0.0)$ & $47.59( \pm 0.1)$ & $47.97( \pm 0.1)$ \\
Rolls & $45.89( \pm 0.2)$ & $4.51( \pm 0.1)$ & $49.60( \pm 0.4)$ & $54.11( \pm 0.3)$ \\
Dhal wade & $44.11( \pm 0.3)$ & $0.91( \pm 0.0)$ & $54.97( \pm 0.5)$ & $55.88( \pm 0.3)$ \\
Ulundu wade & $51.92( \pm 0.2)$ & $0.00( \pm 0.0)$ & $48.08( \pm 0.7)$ & $48.08( \pm 0.5)$ \\
Prawn wade & $43.17( \pm 0.2)$ & $1.37( \pm 0.0)$ & $55.46( \pm 0.6)$ & $56.83( \pm 0.3)$ \\
Samosa & $47.00( \pm 0.3)$ & $5.99( \pm 0.05)$ & $47.00( \pm 0.5)$ & $52.99( \pm 0.4)$ \\
Patties & $20.63( \pm 0.1)$ & $32.59( \pm 0.2)$ & $40.21( \pm 0.1)$ & $72.80( \pm 0.2)$ \\
Curry rotti & $46.27( \pm 0.4)$ & $16.45( \pm 0.2)$ & $37.28( \pm 0.2)$ & $53.73( \pm 0.2)$ \\
Murukku & $40.46( \pm 0.3)$ & $5.52( \pm 0.2)$ & $54.02( \pm 0.8)$ & $59.54( \pm 0.6)$ \\
Manioc chips & $44.92( \pm 0.3)$ & $5.20( \pm 0.05)$ & $49.88( \pm 0.7)$ & $55.08( \pm 0.55)$ \\
Dodol & $77.44( \pm 0.2)$ & $0.00( \pm 0.0)$ & $22.56( \pm 0.1)$ & $22.56( \pm 0.1)$ \\
Unduwel & $48.84( \pm 0.3)$ & $1.59( \pm 0.1)$ & $49.57( \pm 0.5)$ & $51.16( \pm 0.4)$ \\
Kadala & $54.98( \pm 0.5)$ & $5.55( \pm 0.05)$ & $39.47( \pm 0.3)$ & $45.02( \pm 0.25)$ \\
Popcorn & $54.19( \pm 0.6)$ & $3.30( \pm 0.02)$ & $42.51( \pm 0.2)$ & $45.81( \pm 0.15)$ \\
\hline
\end{tabular}

SFA - Saturated fatty acid; MUFA - Monounsaturated fatty acid; PUFA - Polyunsaturated fatty acid; UFA - Unsaturated fatty acid. 
However, in our studies no such high levels of trans fat were detected in bakery foods. Ruminant products such as beef, milk, cheese, butter, yoghurt and curd contain up to about $2.5 \%$ trans fat which are generated during the process known as biohydrogenation. However, these fatty acids are different from industrially produced transfatty acids, therefore, those fatty acids are considered separately. In fact, some of the trans fatty acids produced through biohydrogenation carry bioactive properties such as anticancer activity. In the present study, ruminant products were not tested. Of the two fatty acids industrially generated trans fatty acid, elaidic acid is the major trans fatty acid formed during processing. Tables 5 and 6 present the quantities of elaidic acid and linolelaidic acid found in the samples. Elaidic acid (C18:1 trans-9) is the predominant trans fatty acid found in most of food samples such as fried whole chicken, chicken nuggets, fried whole fish, chilli paste, french fries, manioc chips (foods collected

Table 5. Mean $( \pm S D, n=3)$ of elaidic acid (C18:1t) and linolelaidic acid (C18:2t) content in foods collected from restaurants and bakeries

\begin{tabular}{lcc}
\hline Food Item & $\begin{array}{c}\text { Elaidic acid (C18:1t) } \\
\text { (g/100 g food) }\end{array}$ & $\begin{array}{c}\text { Linolelaidic acid (C18:2t) } \\
\text { (g/100 g food) }\end{array}$ \\
\hline Fried Whole chicken & $0.08( \pm 0.0)$ & $0.00( \pm 0.0)$ \\
Chicken nuggets & $0.02( \pm 0.0)$ & $0.00( \pm 0.0)$ \\
Fried whole fish & $0.04( \pm 0.0)$ & $0.02( \pm 0.0)$ \\
Chilli paste & $1.32( \pm 0.02)$ & $0.25( \pm 0.0)$ \\
French fries & $0.03( \pm 0.0)$ & $0.00( \pm 0.0)$ \\
Manioc chips & $0.91( \pm 0.01)$ & $0.04( \pm 0.0)$ \\
Popcorn & $0.00( \pm 0.0)$ & $0.37( \pm 0.0)$ \\
Fried rice & $0.00( \pm 0.0)$ & $0.00( \pm 0.0)$ \\
Kottu & $0.00( \pm 0.0)$ & $0.00( \pm 0.0)$ \\
Pizza & $0.00( \pm 0.0)$ & $0.00( \pm 0.0)$ \\
White bread & $0.00( \pm 0.0)$ & $0.00( \pm 0.0)$ \\
Sandwich bread & $0.00( \pm 0.0)$ & $0.00( \pm 0.0)$ \\
Parata & $0.00( \pm 0.0)$ & $0.00( \pm 0.0)$ \\
Poori & $0.00( \pm 0.0)$ & $0.00( \pm 0.0)$ \\
\hline
\end{tabular}

Table 6. Mean $( \pm S D, n=3)$ of elaidic acid (18:1t) and linolelaidic acid (18:2t) content in street foods.

\begin{tabular}{lcc}
\hline Food Item & $\begin{array}{c}\text { Elaidic acid (18:1t) } \\
\text { (g/100 g food) }\end{array}$ & $\begin{array}{c}\text { Linolelaidic acid (18:2t) } \\
\text { (g/100 g food) }\end{array}$ \\
\hline Fried rice & $0.00( \pm 0.0)$ & $0.00( \pm 0.0)$ \\
Kottu & $0.00( \pm 0.0)$ & $0.00( \pm 0.0)$ \\
Cutlets & $0.00( \pm 0.0)$ & $0.00( \pm 0.0)$ \\
Rolls & $0.00( \pm 0.0)$ & $0.00( \pm 0.0)$ \\
Dhal wade & $0.00( \pm 0.0)$ & $0.00( \pm 0.0)$ \\
Ulundu wade & $0.00( \pm 0.0)$ & $0.00( \pm 0.0)$ \\
Prawn wade & $0.00( \pm 0.0)$ & $0.00( \pm 0.0)$ \\
Samosa & $0.15( \pm 0.03)$ & $0.00( \pm 0.0)$ \\
Patties & $0.05( \pm 0.0)$ & $0.04( \pm 0.0)$ \\
Curry rotti & $0.09( \pm 0.0)$ & $0.02( \pm 0.0)$ \\
Murukku & $0.15( \pm 0.01)$ & $0.00( \pm 0.0)$ \\
Manioc chips & $0.10( \pm 0.03)$ & $0.07( \pm 0.0)$ \\
Dodol & $0.00( \pm 0.0)$ & $0.00( \pm 0.0)$ \\
Unduwel & $0.00( \pm 0.0)$ & $0.00( \pm 0.0)$ \\
Kadala & $0.07( \pm 0.0)$ & $0.00( \pm 0.0)$ \\
Popcorn & $0.02( \pm 0.0)$ & $0.00( \pm 0.0)$ \\
\hline
\end{tabular}




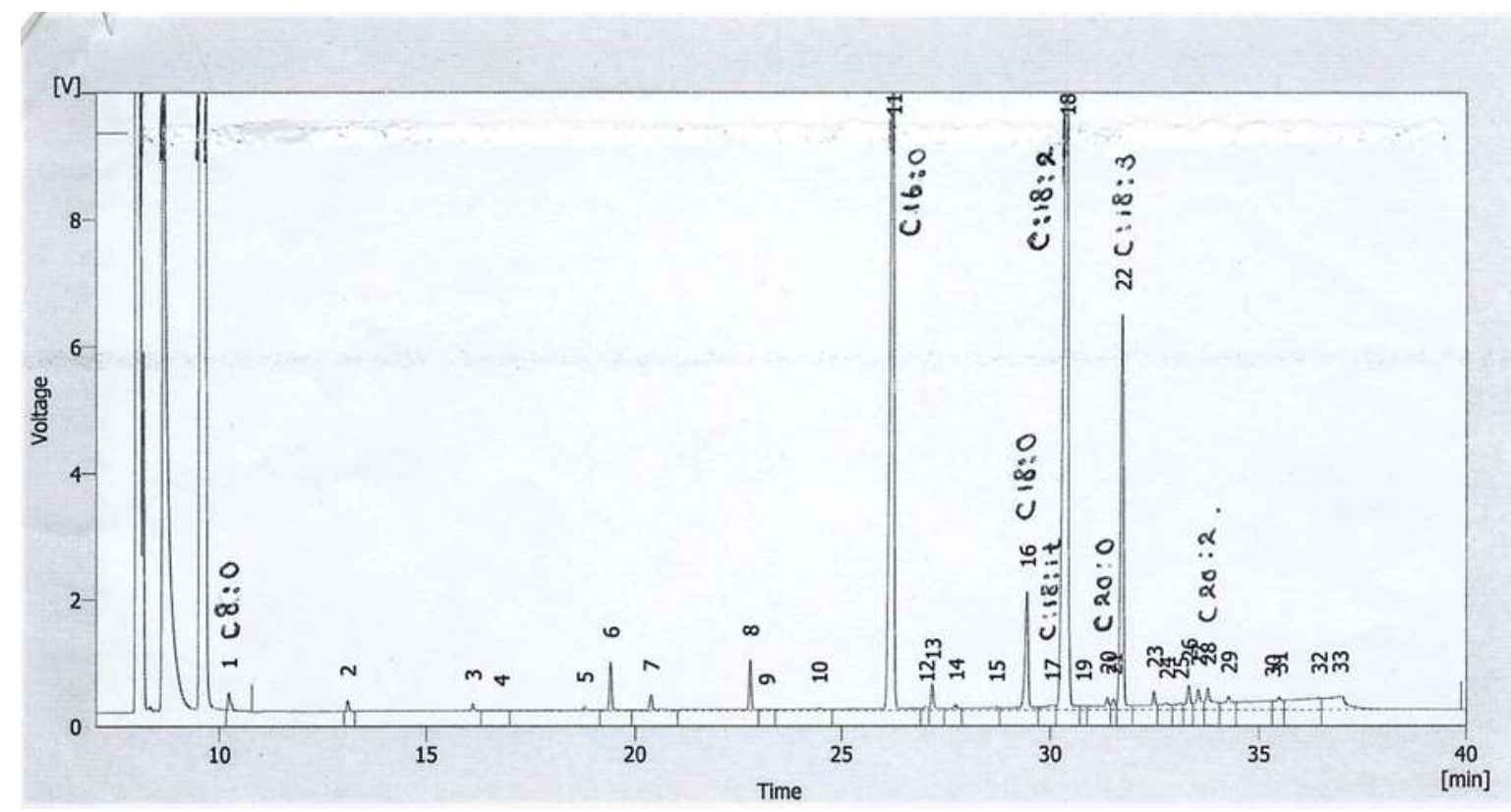

Figure 1. A representative chromatogram illustrating the fatty acids present in chilli paste

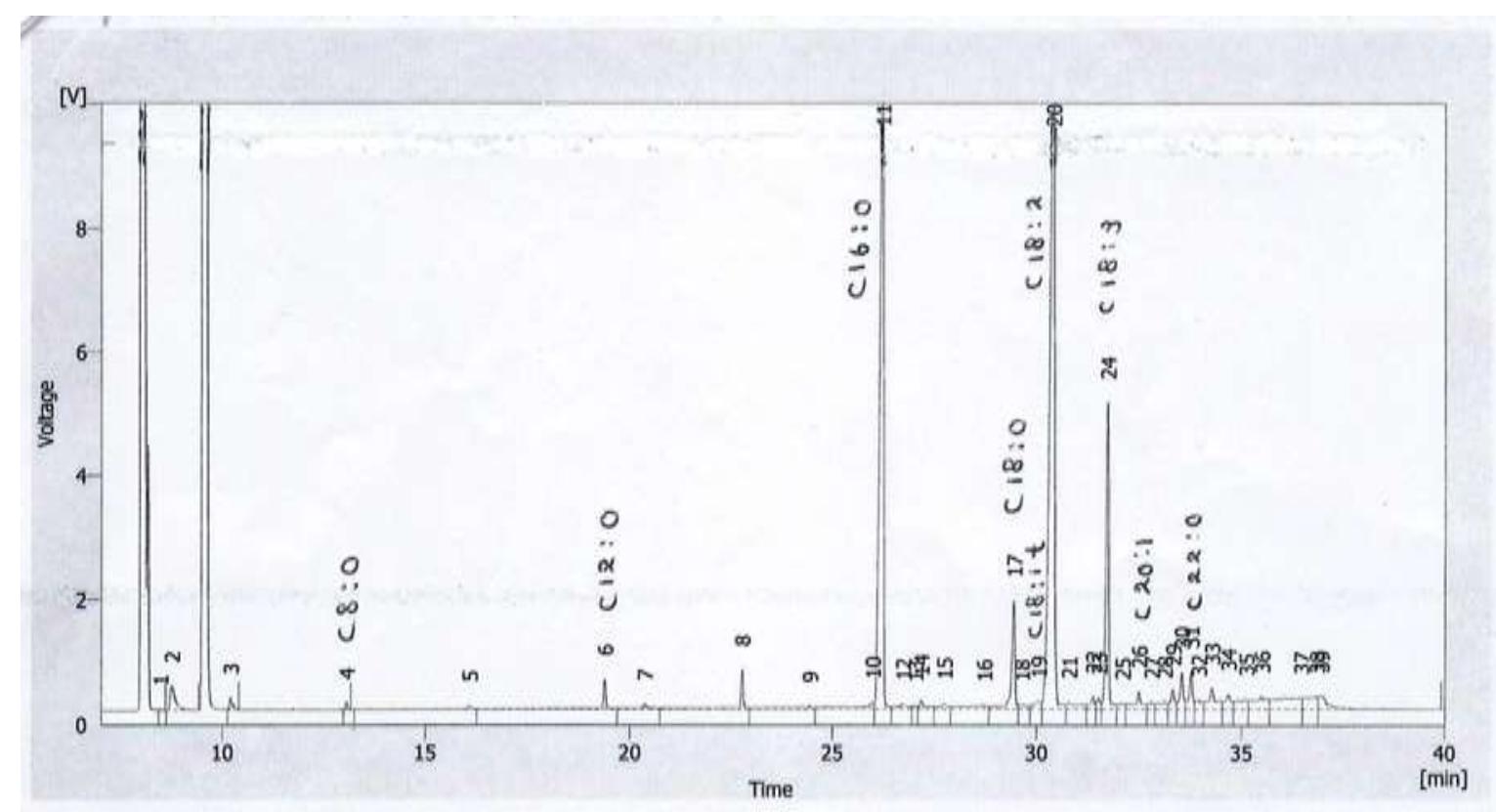

Figure 2. A representative chromatogram illustrating the fatty acids present in manioc chips

from bakeries and restaurants), samosa, patties, curry rottie, murukku, manioc chips, kadala and popcorn (street foods). Linolelaidic acid (C18:2 trans-6) was detected in few food samples such as fried whole fish, chilli paste, manioc chips, popcorn (foods collected from bakeries and restaurants), patties, curry rottie and manioc chips (street foods). According to Chajes et al. (2011), elaidic acid is the main trans fatty acid isomer generated during partial hydrogenation of vegetable oils used as ingredients for the formulation of processed foods. Figures 1 and 2 are representative GLC chromatograms illustrating different fatty acids identified in chilli paste and manioc chips.

Considering the stability of oil during frying, most food manufactures in Sri Lanka tend to use palm oil as the major frying oil. In addition, it is suspected 
that many manufacturers tend to use oil repeatedly in order to cut down the cost of production. Palm oil contains nearly $10 \%$ of polyunsaturated fatty acids thus, there is a possibility of generating TFA during frying at high temperature for long time (Tynek et al., 2001) compared to coconut oil. Furthermore, it is suspected that spent oil which has been used repeatedly for many frying cycles is used in the manufacture of products such as chilli paste and preparation of fried rice and in roasted chicken as a glaze.

Trans fats are mainly generated during partial hydrogenation of liquid vegetable oils, refining and industrial processing. Partial hydrogenation is used primarily for two reasons as convert liquid oils to solids and to improve the oxidative stability of these fats (Uauy et al., 2009).Partial hydrogenation forms a mixture of cis and transfatty acids. Therefore, the use of partial hydrogenation has been restricted in many countries.

The consumption of TFAs is associated with a high risk of coronary heart diseases, cancer and obesity. It has been shown that daily intake of $6 \mathrm{~g}$ TFA can lead to $50 \%$ increase in cardiovascular disease incidences. A regular intake of such fast food products (twice a week or more) over a long period of time may increase the risk of CVD (Karl et al., 1999). The WHO recommends that the TFA should be limited to $1 \%$ of total energy intake.

\section{REFERENCES}

Aladedunye, F.A. and Przybylski, R. (2009). Degradation and nutritional quality changes of oil during frying. Journal of the American Oil Chemists' Society, 86(2), 149-156.

Bhatnagar, A.S., Kumar, P.P., Hemavathy, J. and Krishna, A.G. (2009). Fatty acid composition, oxidative stability, and radical scavenging activity of vegetable oil blends with coconut oil. Journal of the American Oil Chemists' Society, 86(10), 91-999.

Chajès, V., Biessy, C., Byrnes, G., Deharveng, G., Saadatian-Elahi, M., Jenab, M., Peeters, P.H., Ocké, M., Bueno-de-Mesquita, H.B., Johansson, I. and Hallmans, G. (2011). Ecological-level associations between highly processed food intakes and plasma phospholipid elaidic acid concentrations: results from a cross-sectional study within the European prospective investigation into cancer and nutrition (EPIC). Nutrition and Cancer, 63(8), 1235-1250.

\section{CONCLUSIONS}

Among the studied food samples, collected from bakery chains and restaurants, grocery stores and small food outlets from Colombo district, chilli paste and manioc chips contained the highest amount of trans fatty acids. Other food items did not contain considerable amount of trans fatty acids. In general, the foods collected from roadside vendors contained comparatively higher amounts of trans fatty acids. Elaidic acid (C18:1t) and linolelaidic acid (C18:2t) are the main two trans fatty acids detected in the foods tested. Of the two trans fatty acids, elaidic acid was the predominant trans fatty acids detected in foods tested. The saturated fatty acid content of foods prepared using coconut such as Dodol contain high amounts of saturated fatty acids.

\section{ACKNOWLEDGEMENT}

The authors acknowledge the financial assistance provided by the World Health Organization and the administrative assistance provided by the Agribusiness Centre, Faculty of Agriculture, University of Peradeniya.

De Souza, R.J., Mente, A., Maroleanu, A., Cozma, A.I., Ha, V., Kishibe, T., Uleryk, E., Budylowski, P., Schünemann, H., Beyene, J. and Anand, S.S. (2015). Intake of saturated and trans unsaturated fatty acids and risk of all cause mortality, cardiovascular disease, and type 2 diabetes: systematic review and meta-analysis of observational studies. Bmj, 351.

Dhaka, V., Gulia, N., Ahlawat, K.S. and Khatkar, B.S. (2011). Trans fats-sources, health risks and alternative approach-A review. Journal of food science and technology, 48(5), 534-541.

Kaushik, I. and Grewal, R.B. (2017). Trans Fatty Acids: Replacement Technologies in Food. Advances in Research, 9 (5), 1- 14.

Kavanagh, K., Jones, K.L., Sawyer, J., Kelley, K., Carr, J.J., Wagner, J.D. and Rudel, L.L. (2007). Trans fat diet induces abdominal obesity and changes in insulin sensitivity in monkeys. Obesity, 15(7), 1675-1684. 
Khosla, P. (2006). Palm Oil: A nutritional overview. Agro Food Industry Hi Tech, 17(3), 21.

Madhujith, T. and Sivakanthan, S. (2020). Taking Trans fats out of the food supply. Agricultural research for sustainable food systems in Sri Lanka, 277-299.

Martinet, W., Knaapen, M. W., Kockx, M. M. and De Meyer, G. R. (2007). Autophagy in cardiovascular disease. Trends in molecular medicine, 13(11), 482-491.

Paquot, C. and Hautfenne, A. (1979). Standard methods for the analysis of oils, fats and derivatives. Pure and Applied Chemistry, 51, 2503-2526.

Rani, A.K.S., Reddy, S.Y. and Chetana, R. (2010). Quality changes in trans and trans free fats/oils and products during frying. European food research and technology, 230(6), 803-811.

Ratnayake, W.M.N. (2004). Overview of methods for the determination of trans fatty acids by gas chromatography, silver-ion thin-layer chromatography, silver-ion liquid chromatography, and gas chromatography/mass spectrometry. Journal of AOAC International, 87(2), 523-539.

Remig, V., Franklin, B., Margolis, S., Kostas, G., Nece, T. and Street, J.C. (2010). Trans fats in America: a review of their use, consumption, health implications, and regulation. Journal of the American Dietetic Association, 110(4), 585592.

Tynek, M., Hazuka, Z., Pawlowicz, R. and Dudek, M. (2001). Changes in the frying medium during deep-frying of food rich in proteins and carbohydrates. Journal of food Lipids, 8(4), 251-261.

Uauy, R., Aro, A., Clarke, R., L'abbé, M.R., Mozaffarian, D., Skeaff, C.M., Stender, S. and Tavella, M. (2009). WHO Scientific Update on trans fatty acids: summary and conclusions. European Journal of Clinical Nutrition, 63(2), S68-S75.

Wanders, A., Zock, P. and Brouwer, I. (2017). Trans fat intake and its dietary sources in general populations worldwide: a systematic review. Nutrients, 9(8), 840. 